\title{
A Multicenter Study on the Prevalence of Eosinophilic Esophagitis and PPI-Responsive Esophageal Eosinophilic Infiltration
}

\author{
Yasuhiro Fujiwara ${ }^{1}$, Takashi Sugawa ${ }^{2}$, Fumio Tanaka ${ }^{3}$, Hiroshi Tatsuwaki ${ }^{4}$, \\ Masatsugu Okuyama ${ }^{5}$, Tsuyoshi Hayakawa ${ }^{6}$, Kazuki Yamamori ${ }^{2}$, Rikimon Wada ${ }^{7}$, \\ Kenjiro Ohtani ${ }^{8}$, Hironori Uno ${ }^{9}$, Tetsuya Tanigawa ${ }^{1}$, Yoshihisa Watanabe ${ }^{7}$, \\ Kazunari Tominaga ${ }^{1}$, Toshio Watanabe ${ }^{1}$, Osamu Takaishi ${ }^{5}$, Yoshihiko Saeki ${ }^{6}$, \\ Hiroko Nebiki ${ }^{10}$, Nobuhide Oshitani ${ }^{3}$, Hiroshi Sato ${ }^{10}$ and Tetsuo Arakawa ${ }^{1}$
}

\begin{abstract}
Objective Eosinophilic esophagitis (EoE) is diagnosed by the presence of dysphagia and intraepithelial eosinophilic infiltration of $\geq 15$ per high-power field (HPF). EoE should be distinguished from proton pump inhibitor-responsive esophageal eosinophilic infiltration (PPI-R EEI) in patients that are responsive to PPI treatment. The aim of this study was to determine the prevalence of EoE and PPI-R EEI in Japanese patients in a multicenter study.

Methods Ten hospitals participated in this study. Esophageal biopsy was performed when the patients had typical EoE symptoms or when endoscopic findings revealed a typical EoE appearance. EEI was defined as the intraepithelial eosinophilic infiltration of $\geq 15$ per HPF. Patients with EEI received rabeprazole for 8 weeks to distinguish EoE from PPI-R EEI.

Results A total of 13,634 subjects that underwent upper gastrointestinal endoscopy because of further examination or as a routine checkup were enrolled. Seventy-one $(0.5 \%)$ patients suspected with EoE were examined by biopsy. A histological examination of 7 (9.9\%) cases revealed EEI. Two of these 7 patients showed no symptoms and the other 5 were treated with PPI. Two $(0.01 \%)$ patients were diagnosed with EoE and $3(0.02 \%)$ with PPI-R EEI.
\end{abstract}

Conclusion EoE and PPI-R EEI were rare in Japanese patients that underwent upper gastrointestinal endoscopy.

Key words: eosinophilic esophagitis, proton pump inhibitor, Japanese, epidemiology

(Intern Med 51: 3235-3239, 2012)

(DOI: 10.2169/internalmedicine.51.8670)

\section{Introducion}

Esophageal eosinophilic infiltration (EEI) is observed in several conditions, including gastroesophageal reflux disease (GERD), eosinophilic esophagitis (EoE), celiac disease, infections, Crohn's disease, achalasia, drug hypersensitivity, vasculitis, and graft-versus-host disease $(1,2)$. Eosinophilic

\footnotetext{
${ }^{1}$ Department of Gastroenterology, Osaka City University Graduate School of Medicine, Japan, ${ }^{2}$ Department of Gastroenterology, Osaka Hospital of Japanese Seafarers Relief Association, Japan, ${ }^{3}$ Department of Gastroenterology, Izumiotsu Municipal Hospital, Japan, ${ }^{4}$ Department of Internal Medicine, Yamamoto Third Hospital, Japan, ${ }^{5}$ Department of Gastroenterology, Kashiwara Municipal Hospital, Japan, ${ }^{6}$ Department of Gastroenterology, Imazato Gastrointestinal Hospital, Japan, ${ }^{7}$ Department of Gastroenterology, Asakayama General Hospital, Japan, ${ }^{8}$ Department of Gastroenterology, Nagayoshi General Hospital, Japan, ${ }^{9}$ Department of Gastroenterology, Ohno Memorial Hospital, Japan and ${ }^{10}$ Department of Gastroenterology, Osaka City General Hospital, Japan
}

Received for publication July 25, 2012; Accepted for publication September 2, 2012

Correspondence to Dr. Yasuhiro Fujiwara, yasu@med.osaka-cu.ac.jp 
esophagitis (EoE) is recently recognized as a chronic, immune/antigen-mediated esophageal disease (2). The American Gastroenterological Association (AGA) recommends that EoE be diagnosed by the presence of typical symptoms such as dysphagia and food impaction, and intraepithelial eosinophilic infiltration of $\geq 15$ per high-power field (HPF) (1). The AGA recommendations state that EoE should be distinguished from other causes of EEI, especially GERD (1). However, the association between GERD and EoE is controversial because gastric acid might play a role in the pathogenesis of EoE (3) and proton pump inhibitors (PPIs) are effective in some cases of EoE (4). The updated consensus and recommendation by the American Academy of Allergy, Asthma, and Immunology reported that PPI-responsive EEI (PPI-R EEI) is indicated when administration of PPI improves symptoms and eosinophilic infiltration in cases with similar clinical characteristics of EoE (2).

The prevalence of EoE in the US is estimated to be $0.44 \%$ among adults (5). A prospective study carried out in 1,000 patients in Sweden estimated the prevalence at almost $1 \%$ (6). Several epidemiological studies have been conducted in Western countries (5-10), but the prevalence of EoE may vary depending on different factors, eg, the geographical area or the targeted population. Most Western studies show a significant increase in the rate of $\operatorname{EoE}(7,8)$. However, there are only a few studies on EoE conducted in Asia, including Japan (11-15). The purpose of this study was to determine the prevalence of EoE and PPI-R EEI in Japanese adult patients in a multicenter study.

\section{Materials and Methods}

\section{Subjects}

Patients that underwent upper gastrointestinal (GI) endoscopy for the examination of upper GI symptoms and had abnormal findings detected by other modalities or a routine healthy checkup at 10 hospitals were prospectively enrolled between September 2010 and March 2011. These 10 hospitals were located in Osaka, the third largest prefecture in Japan, and all physicians that participated in this study were gastroenterologists as well as GI endoscopists. Patients less than 18 years of age and those that underwent upper GI endoscopic treatment such as endoscopic mucosal dissection for superficial cancer, endoscopy because of treatments such as variceal ligation and endoscopic hemostasis were excluded. Patients that received acid suppressive drugs, including PPI, were not excluded. This study was approved by the Ethics Committee of the Osaka City University Graduate School of Medicine.

\section{Endoscopic assessment}

EoE findings, reflux esophagitis, and hiatal hernia were diagnosed endoscopically. The endoscopic diagnosis of EoE was based on the finding of (1) mucosal rings defined as transient contractions or fixed structures; (2) linear furrows representative of mucosal edema and thickening with vertical lines along the length of the esophageal mucosa; (3) white exudates scattered across the mucosal surface, which appear as punctate white nodules, dispersant flocculants material, or in a granular pattern; (4) stricture defined as narrowing of the esophageal lumen, resulting in difficulty of passage of the endoscope; and (5) mucosal tears defined as a breach of the esophageal mucosa spontaneously or by air inhalation during endoscopic examination $(1,2)$. Reflux esophagitis was diagnosed and graded according to the Los Angeles classification (grade A-D) (16). This study excluded grade $\mathrm{M}$ (minimal change) because of low interobserver and intraobserver agreements (17). Hiatal hernia was defined as an apparent separation of the esophagogastric junction and diaphragm impression by more than $2 \mathrm{~cm}$ at endoscopy.

\section{Biopsy examination}

Biopsy specimens were obtained from the distal esophagus, proximal esophagus, or the area of the EoE endoscopic findings in patients presenting with typical EoE symptoms or with endoscopic findings of EoE. The number of biopsies depended on each case and the endoscopist. No biopsy was obtained if the patients received antiplatelet or anticoagulant therapy, or had a bleeding tendency for any reasons. Biopsy specimens were fixed in formalin and samples were stained with hematoxylin-eosin. The number of eosinophils infiltrated into the esophageal epithelium was counted. Cases were diagnosed to have EEI if the number of intraepithelial eosinophils was $\geq 15$ in $1 \operatorname{HPF}(1,2)$.

\section{Treatment protocol and definition}

Symptomatic patients with EEI received rabeprazole 10 $\mathrm{mg}$ once daily for 8 weeks. Their symptoms and findings after upper GI endoscopy with a biopsy were re-evaluated after treatment. PPI-R EEI was defined as a case in which administration of PPI improved both symptoms and intraepithelial eosinophilic infiltration $(<15 / \mathrm{HPF})$; EoE was defined as a case with no improvement of symptoms and eosinophilic infiltration (2).

\section{Results}

13,634 patients who underwent upper GI endoscopy at 10 institutes were enrolled. Seventy-one of those $(0.52 \%)$ patients underwent pathological examination by biopsy because of the suspicion of EoE. Forty-three of those patients were men and 28 were women, and their mean age was 58.3 years. The reasons for the suspicion of EoE were the presence of dysphagia or food impaction in $22(31 \%)$ cases, and the endoscopic appearance of EoE in 68 (96\%) cases, including linear furrows in $25(35 \%)$, rings in $16(23 \%)$, and white exudates in $37(52 \%)$. There was no case with stricture and mucosal tearing in this study. An examination of the biopsy specimens identified 7 cases as EEI defined as intraepithelial eosinophilic infiltration of $\geq 15$ per HPF.

The clinical characteristics of 7 cases with EEI are shown 
Table. Clinical Characteristics of Cases with Esophageal Eosinophilic Infiltration

\begin{tabular}{|c|c|c|c|c|c|c|c|c|c|c|c|c|c|c|c|}
\hline \multirow{2}{*}{\multicolumn{2}{|c|}{ Age }} & \multirow[t]{2}{*}{ Sex } & \multirow{2}{*}{$\begin{array}{l}\text { Smoking } \\
\text { habits }\end{array}$} & \multirow{2}{*}{$\begin{array}{l}\text { Drinking } \\
\text { habits }\end{array}$} & \multicolumn{2}{|c|}{ Symptoms } & \multirow{2}{*}{ Allergy } & \multicolumn{3}{|c|}{ Endoscopic findings } & \multirow[b]{2}{*}{$\begin{array}{l}\text { Histology } \\
\text { Number } \\
\text { of } \\
\text { eosinophils } \\
\text { (per } \\
\text { HPF) }\end{array}$} & \multirow{2}{*}{$\begin{array}{l}\text { Peripheral } \\
\text { eosinophils } \\
\qquad / \mu \mathrm{L})\end{array}$} & \multirow{2}{*}{$\begin{array}{c}\text { T-IgE } \\
(\mathrm{IU} / \mathrm{m} \\
\mathrm{L})\end{array}$} & \multirow{2}{*}{$\begin{array}{c}H . \\
\text { pylori } \\
\text { infecti } \\
\text { on }\end{array}$} & \multirow{2}{*}{$\begin{array}{c}\text { PPI } \\
\text { response }\end{array}$} \\
\hline & & & & & $\begin{array}{l}\text { Dysphagia/ } \\
\text { Food } \\
\text { impaction }\end{array}$ & Heartburn & & $\begin{array}{l}\text { EoE-like } \\
\text { appearance }\end{array}$ & $\begin{array}{l}\text { Mucosal } \\
\text { break } \\
\text { (Grade) }\end{array}$ & $\begin{array}{l}\text { Hiatal } \\
\text { hernia }\end{array}$ & & & & & \\
\hline 1 & 37 & $\mathrm{M}$ & - & + & + & - & + & F, R, W & - & - & 25 & 289 & 180 & negative & No \\
\hline 2 & 70 & M & + & + & + & - & - & $\mathrm{W}$ & B & + & 20 & 196 & 14 & negative & Yes \\
\hline 3 & 47 & $\mathrm{M}$ & - & - & - & + & + & $\mathrm{F}, \mathrm{R}$ & B & - & 22 & 433 & 5 & negative & Yes \\
\hline 4 & 50 & $\mathrm{M}$ & + & + & - & + & + & $\mathrm{F}$ & A & - & 40 & NT & NT & NT & NT \\
\hline 5 & 50 & $\mathrm{M}$ & - & - & - & - & - & $\mathrm{F}, \mathrm{R}$ & A & - & 18 & NT & NT & NT & NT \\
\hline 6 & 61 & $\mathrm{M}$ & - & + & - & + & + & $\mathrm{F}$ & - & + & 30 & 112 & 890 & negative & Yes \\
\hline 7 & 37 & $\mathrm{M}$ & - & + & + & - & - & $\mathrm{F}, \mathrm{W}$ & B & - & 106 & NT & NT & negative & No \\
\hline
\end{tabular}

EoE: eosinophilic esophagitis, HPF: high-power field, PPI: proton pump inhibitor, F: linear furrows, R: ring, W: white exudates, NT: not tested

Total cases of upper $\mathrm{Gl}$ endoscopy 13,634 cases

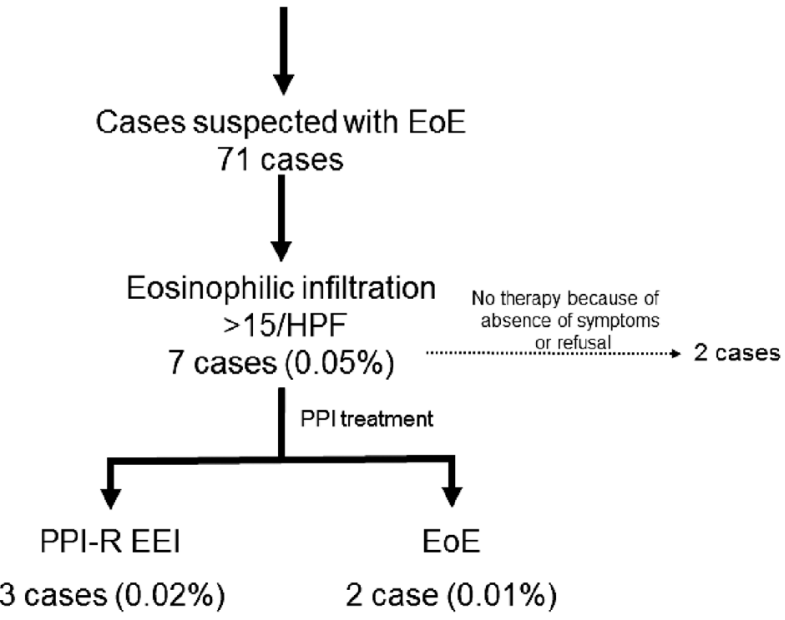

Figure 1. Prevalence of eosinophilic esophagitis (EoE) and proton pump inhibitor -responsive esophageal eosinophilic infiltration (PPI-R EEI). A total of 13,634 patients that underwent upper gastrointestinal (GI) endoscopy were enrolled. Seven of the 71 patients suspected to have EoE were diagnosed as having EEI defined as intraepithelial infiltration of $\geq 15$ per high-power field (HPF). Two of those patients had no symptoms and the other 5 patients received rabeprazole for 8 weeks. Finally, $2(0.01 \%)$ cases with $\mathrm{EoE}$ and $3(0.02 \%)$ cases with PPI-R EEI were identified.

in Table. They were all men and their age ranged from 37 to 70 years, with a mean age of 50.3 years. Two patients were smokers and 5 drinkers. Five $(71 \%)$ patients were symptomatic, including 3 with dysphagia or food impaction. Four $(57 \%)$ patients had allergic diseases, including atopic dermatitis in 1 case, pollinosis in 2, and allergy rhinitis in 1 . None of the patients had a family history of EoE. Endoscopic findings demonstrated linear furrows in 6 cases, ring in 3 , and white exudates in 1 . Five $(71 \%)$ patients had reflux esophagitis and 2 had hiatal hernia. The number of infiltrated intraepithelial eosinophils ranged from 18 to 160 per HPF. Five patients had no Helicobacter pylori infection while two cases were not tested. No increase in peripheral eosinophil counts was found and total IgE was increased in $1(25 \%)$ of 4 cases. The status of 2 of the 5 symptomatic patients with EEI did not improve after PPI therapy, identifying them as having EoE, while the status of the other 3 patients improved after PPI therapy, resulting in the diagnosis of PPI-R EEI. Two $(0.01 \%)$ and $3(0.02 \%)$ of the 13,634 patients that underwent upper GI endoscopy were diagnosed as having EoE and PPI-R EEI, respectively (Fig. 1). The typical endoscopic appearance and histology of the biopsied specimens of EoE (case 1) are shown in Fig. 2. One case (case 1) with EoE received fluticasone swallowing therapy and his symptoms and esophageal eosinophil infiltration were improved. Another case (case 7) with EoE was followed-up because of mild symptoms.

\section{Discussion}

The present multicenter study demonstrated that the prevalence of EEI defined as intraepithelial eosinophilic infiltration of $\geq 15$ per HPF was $7(0.05 \%)$ of 13,634 Japanese patients that underwent upper GI endoscopy. These included 2 cases with EoE and 3 cases with PPI-R EEI. These results suggest that EoE and PPI-R EEI are rare in Japanese adults, revealing a prevalence of $0.01 \%$ and $0.02 \%$, respectively.

Although there are many studies of EoE from Western counties (5-10), there are only a few case reports or series of adult EoE in Asia (11-15). Only 1 case of an asymptomatic Chinese patient with EoE was reported in 2008 (12). Furuta et al. reported the first case of a Japanese patient with EoE in 2006 (11), and several cases of symptomatic or asymptomatic EEI, including EoE and PPI-R EEI, were reported $(13,14)$. Fujishiro et al. conducted a large epidemiological study of EoE (15). They examined 23,346 patients that had undergone upper GI endoscopy and found that 4 patients were endoscopically and histologically diagnosed as having EoE, suggesting that the estimated prevalence of EoE was $17.1 / 100,000(15)$. They stated that the prevalence in 


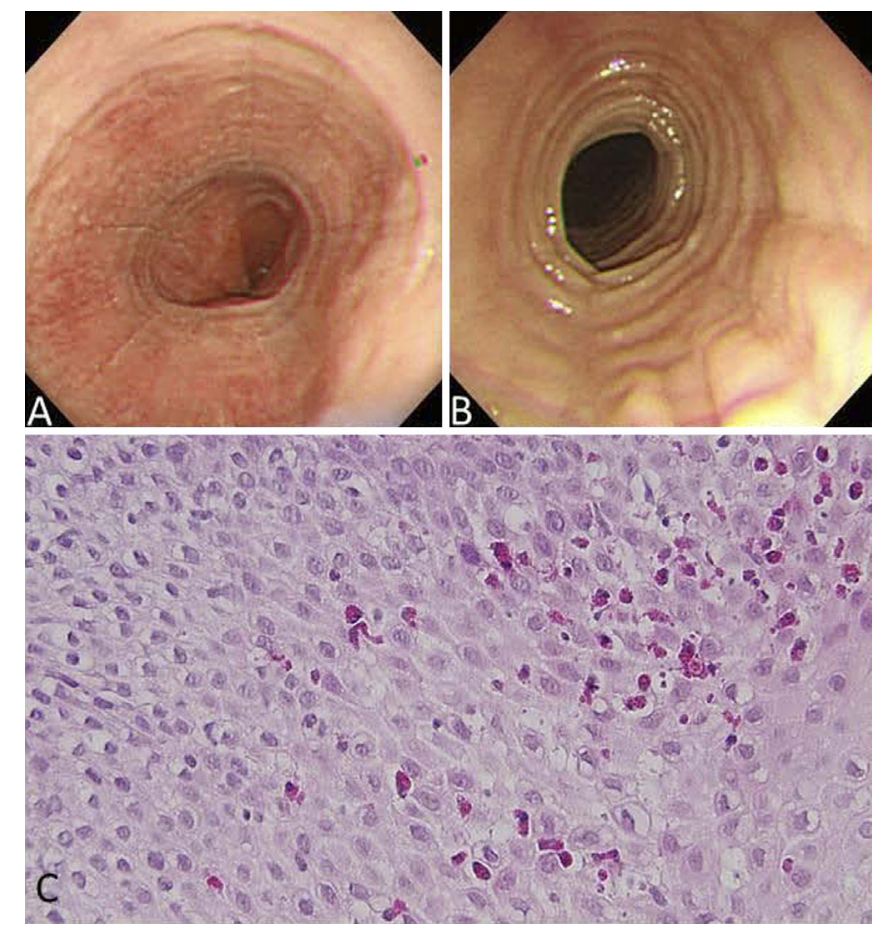

Figure 2. Typical endoscopic appearance and histology of eosinophilic esophagitis (EoE; case 1). The endoscopic image shows linear furrows with a few white exudates in the distal esophagus (A) and a ring at the proximal esophagus (B). Histology of the biopsy specimen revealed marked intraepithelial eosinophilic infiltration $(\mathbf{C})$.

Japanese patients was similar to that in Swiss patients (23/ $100,000)$ (9) as well as that in US patients $(55 / 100,000)(7)$. However, Fujishiro's study included asymptomatic and PPIresponsive cases as EoE. Therefore, definitive EoE was only diagnosed in $1(0.004 \%)$ of 23,346 cases, thus suggesting that EoE is a rare disease in Japan, which was confirmed in the present study.

Molina-Infante et al. examined 712 patients with upper GI symptoms by endoscopy, biopsy, and esophageal $\mathrm{pH}$ monitoring (18). They identified 35 (4.9\%) patients with intraepithelial eosinophilic infiltration of $\geq 15 / \mathrm{HPF}$. Nine $(25.7 \%)$ of the 35 patients were finally diagnosed as having EoE, including 5 cases with concomitant GERD, and 26 (74.3\%) as PPI-R EEI (18). Abe et al. published a Japanese case series of 12 patients with EEI. They identified 2 (28.6\%) cases with EoE and 5 (71.4\%) cases with PPI-R EEI among 7 patients that received PPI (14). Although PPI-R EEI was more common than EoE among patients with EEI, the present study identified 2 cases with EoE and 3 with PPI-R EEI. This might be related to the sample size of EEI in this study.

The clinical characteristics of EoE patients in Western countries revealed a middle age and male predominance, higher comorbidity of allergic diseases, and family aggregation $(1,2)$. Similarly, the current study showed that all cases with EoE and PPI-R EEI were middle-aged men and half of them had allergic diseases, but no family aggregation. There is still no report of family aggregation of EoE in Asia, including Japan. All cases, other than two that were not tested, were found to be $H$. pylori negative. This is consistent with the recent large cross-sectional analysis that showed that $H$. pylori infection was inversely associated with esophageal eosinophilia (19).

The reasons for the low prevalence of EoE and PPI-R EEI in Japan are unknown. Racial differences might be the most important factor. In addition, the pathogenesis of PPI$\mathrm{R}$ EEI is largely unknown at present. Two of the 3 cases with PPI-R EEI had erosive esophagitis. This finding suggests that gastric acid reflux might be associated with similar characteristics of EoE, especially the endoscopic appearance and symptoms.

The present study has some potential limitations. First, the recognition of EoE and PPI-R EEI in Japan is still low, although a meeting presenting the study protocol was held to provide information about the definition and diagnosis of EoE. It is possible that some cases were misdiagnosed, especially cases with a normal endoscopic appearance. Second, the present multicenter study was conducted in departments of gastroenterology and endoscopic units, thus the diagnosis of EoE was mostly based on the endoscopic appearance. A recent study suggests that physicians prefer endoscopic findings for the diagnosis of EoE, even in Western countries (20). Third, the number of biopsy specimens and the locations from where they are obtained are still controversial (21). Although EEI was defined according to the AGA guidelines, a recent study demonstrated that higher cutoff values are useful for the diagnosis of EoE. Further studies on this subject are needed (18).

In conclusion, the prevalence of EoE and PPI-R EEI was $2(0.01 \%)$ and $3(0.02 \%)$ of the 13,634 patients that underwent upper GI endoscopy, respectively. These results suggest that EoE and PPI-R EEI are rare diseases in Japan.

\section{Author's disclosure of potential Conflicts of Interest (COI).}

Arakawa T: Employment/Leadership position/Advisory role, Otsuka Pharmaceutical and Eisai; Honoraria, Otsuka Pharmaceutical and Eisai; Research funding, Otsuka Pharmaceutical and Eisai. Fujiwara Y: Honoraria, Eisai.

\section{References}

1. Furuta GT, Liacouras CA, Collins MH, et al. Eosinophilic esophagitis in children and adults: a systematic review and consensus recommendations for diagnosis and treatment. Gastroenterology 133: 1342-1363, 2007.

2. Liacouras CA, Furuta GT, Hirano I, et al. Eosinophilic esophagitis: updated consensus recommendations for children and adults. $\mathrm{J}$ Allergy Clin Immunol 128: 3-20, 2011.

3. Rothenberg ME. Biology and treatment of eosinophilic esophagitis. Gastroenterology 137: 1238-1249, 2009.

4. Peterson KA, Thomas KL, Hilden K, Emerson LL, Wills JC, Fang JC. Comparison of esomeprazole to aerosolized, swallowed fluticasone for eosinophilic esophagitis. Dig Dis Sci 55: 1313-1319, 2010.

5. Kapel RC, Miller JK, Torres C, Aksoy S, Lash R, Katzka DA. 
Eosinophilic esophagitis: a prevalent disease in the United States that affects all age groups. Gastroenterology 134: 1316-1321, 2008.

6. Ronkainen J, Talley NJ, Aro P, et al. Prevalence of oesophageal eosinophils and eosinophilic oesophagitis in adults: the population-based Kalixanda study. Gut 56: 615-620, 2007.

7. Prasad GA, Alexander JA, Schleck CD, et al. Epidemiology of eosinophilic esophagitis over three decades in Olmsted County, Minnesota. Clin Gastroenterol Hepatol 7: 1055-1061, 2009.

8. Whitney-Miller CL, Katzka D, Furth EE. Eosinophilic esophagitis: a retrospective review of esophageal biopsy specimens from 1992 to 2004 at an adult academic medical center. Am J Clin Pathol 131: 788-792, 2009.

9. Straumann A, Simon HU. Eosinophilic esophagitis: escalating epidemiology? J Allergy Clin Immunol 115: 418-419, 2005.

10. Sealock RJ, Rendon G, El-Serag HB. Systematic review: the epidemiology of eosinophilic oesophagitis in adults. Aliment Pharmacol Ther 32: 712-719, 2010.

11. Furuta K, Adachi K, Kowari K, et al. A Japanese case of eosinophilic esophagitis. J Gastroenterol 41: 706-710, 2006.

12. Lu HC, Lu CL, Chang FY. Eosinophilic esophagitis in an asymptomatic Chinese. J Chin Med Assoc 71: 362-364, 2008.

13. Sano H, Iwakiri K, Kawami N, et al. Eosinophilic esophagitis: a case report with a review of the literature. Clin J Gastroenterol 3: 279-284, 2010.
14. Abe $\mathrm{Y}$, Iijima K, Ohara $\mathrm{S}$, et al. A Japanese case series of 12 patients with esophageal eosinophilia. J Gastroenterol 46: 25-30, 2011.

15. Fujishiro H, Amano Y, Kushiyama Y, Ishihara S, Kinoshita Y. Eosinophilic esophagitis investigated by upper gastrointestinal endoscopy in Japanese patients. J Gastroenterol 46: 1142-1144, 2011.

16. Lundell LR, Dent J, Bennett JR, et al. Endoscopic assessment of oesophagitis: clinical and functional correlates and further validation of the Los Angeles classification. Gut 45: 172-180, 1999.

17. Amano Y, Ishimura N, Furuta K, et al. Interobserver agreement on classifying endoscopic diagnoses of nonerosive esophagitis. Endoscopy 38: 1032-1035, 2006.

18. Molina-Infante J, Ferrando-Lamana L, Ripoll C, et al. Esophageal eosinophilic infiltration responds to proton pump inhibition in most adults. Clin Gastroenterol Hepatol 9: 110-117, 2011.

19. Dellon ES, Peery AF, Shaheen NJ, et al. Inverse association of esophageal eosinophilia with Helicobacter pylori based on analysis of a US pathology database. Gastroenterology 141: 1586-1592, 2011.

20. Peery AF, Shaheen NJ, Dellon ES. Practice patterns for the evaluation and treatment of eosinophilic oesophagitis. Aliment Pharmacol Ther 32: 1373-1382, 2010.

21. Odze RD. Pathology of eosinophilic esophagitis: What the clinician needs to know. Am J Gastroenterol 104: 485-490, 2009.

(C) 2012 The Japanese Society of Internal Medicine http://www.naika.or.jp/imonline/index.html 